\title{
SOFT ROBOTICS: FLUID-DRIVEN SELF-FOLDING PAPERS
}

\author{
Ha-Ill Chun, Maedeh Mohammadifar and Seokheun Choi*,
}

Bioelectronics \& Microsystems Laboratory, Department of Electrical \& Computer Engineering,

Center for Research in Advanced Sensing Technologies \& Environmental Sustainability (CREATES),

State University of New York at Binghamton, New York, USA

\section{ABSTRACT}

We demonstrate a self-folding paper robot with capillary force driven fluid. When water is sprayed on fluidic channels patterned on paper, the 2-D sheet of paper can be controllably selffolded into various 3-D structures; half-oval, circle, round-edge square, triangle, half-circle, and table. The self-folding paper sheet can be readily fabricated via a double-sided wax printing method, forming a bilayer structure of the fluidic channel and the hydrophobic wax, in which these two layers have different swelling/shrinking properties. The patterned paper performs folding actuation with water and unfolding behavior with evaporation without being mechanically manipulated by external forces or moments. Finally, we create a paper gripper based on this self-folding actuation, conveying a low-weight object. This report demonstrates the possibility of paper microfluidics for selffolding actuation and soft robotics.

\section{INTRODUCTION}

Soft robotics has emerged as a promising field of research to revolutionize wearable, healthcare, and manufacturing applications [1-5]. Such robots offer considerable advantages over traditional hard robots in pursuing open-ended tasks in an unstructured environment and in physical interfaces with living organisms, including humans (NSF EFRI Solicitation 2018). In contrast to their rigid counterparts, soft materials may easily deform and adapt to a wide range of surroundings $[6,7]$. Recently, active soft materials for self-folding robots have attracted significant attention because they inherently convert other forms of energy into mechanical force to create structures without the application of external manipulations [8]. Many self-folding techniques have been developed by using hydraulic, pneumatic, electrical, thermal or magnetic actuation [8-11]. Among those techniques, paper-based soft robotics is the most underdeveloped [12-14]. Even so, excitement is building, as paper possesses unique properties such as low cost, flexible, foldable, eco-friendly, and easily designable. Furthermore, the potential of paper for large-scale batch-fabrication has been demonstrated in recent publications [15]. Recently, the Hashimoto group created a novel method to print an actuator and a structure on a paper substrate [16, 17]. The ink printed on the paper induced the paper substrate to self-fold due to swelling and drying processes. However, this folding process occurred instantly with printing, limiting the ondemand operation and practical applications. Furthermore, this technique had little control of the degree of folding because of the uncontrollable penetration depth of ink in paper. Also, the drying process of the ink induced the paper to undesirably self-fold in the opposite direction.

Here, we introduced an innovative self-folding method with a microfluidic capillary force in paper, allowing on-demand activation with the simple spraying of water (Figure 1). This new technique enabled a flat paper sheet to self-fold with desired angles by controlling wax patterns/thicknesses. The thickness of the hydrophobic wax increased with heating time, reducing the thickness of the hydrophilic part of the paper and controlling the folding degree with water sprayed. This proposed technique will
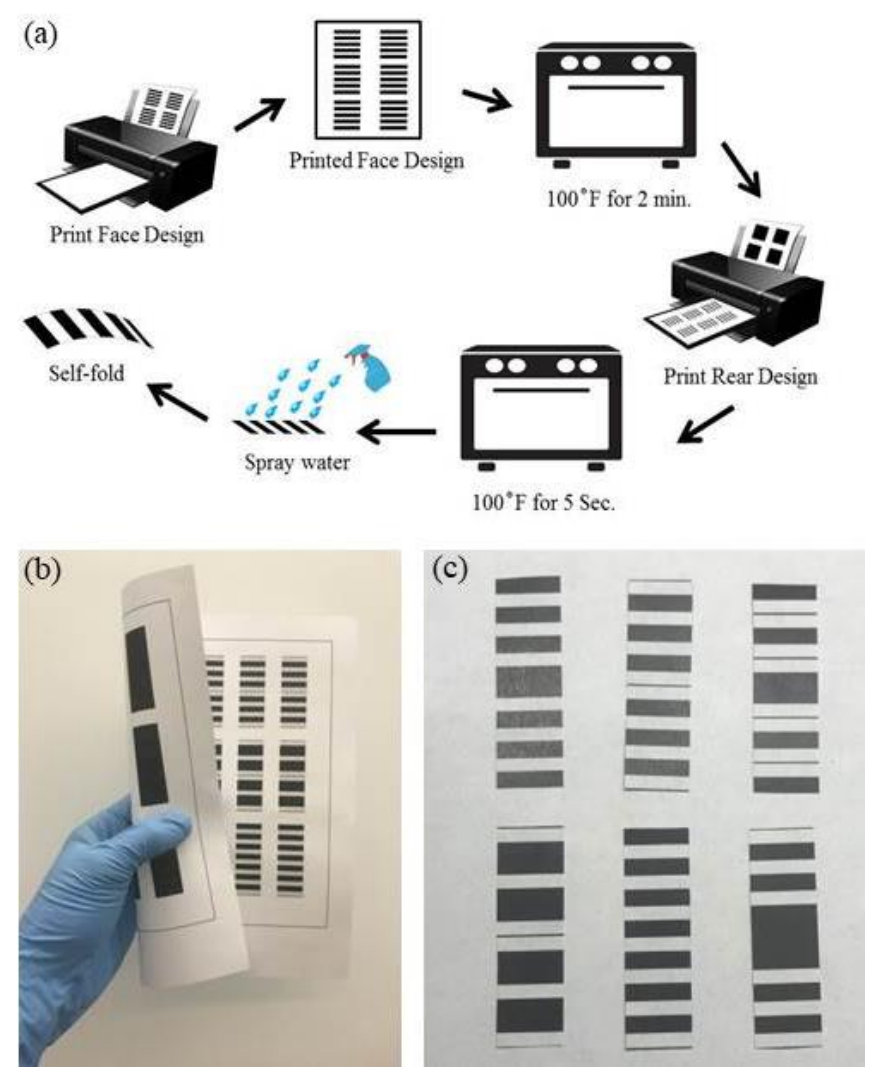

Figure 1: (a) Fabrication processes of the self-folding paper robot. (b) \& (c) Photo-images of the 2-D sheet of papers with different wax patterns.

be advantageous for many applications inexpensively and autonomously in remote and dangerous environments.

\section{MATERIALS AND EXPERIMENTAL SET-UP Device Fabrication}

The hydrophilic channels were defined with hydrophobic wax boundaries on paper by double-sided printing and letting the wax penetrate asymmetrically (Figure 2) [18, 19]. The vertical penetration depth of the melted wax was controlled by adjusting the heating time. Specific wax/channel patterns were designed using AutoCAD software and printed out onto A4 printer papers using a Xerox Phaser printer (ColorQube 8570). The printed wax was heated for various times at $130{ }^{\circ} \mathrm{C}$ (E191047, VWR International, USA), controlling the wax/channel thickness.

\section{Operating Principle}

Figure 2 shows the operating principle of the fluidic actuation in paper, which consists of a swelling process with sprayed water and a drying process with evaporation. This actuation used a twolayer configuration with one passive wax layer and one active water layer. The passive layer developed a negligible mechanical 
(a) A patterned single sheet of paper

\begin{tabular}{|l|}
\hline Hydrophilic channel \\
Hydrophobic wax
\end{tabular}

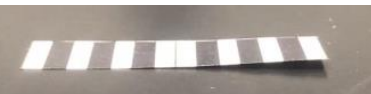

(b) self-folding with sprayed water
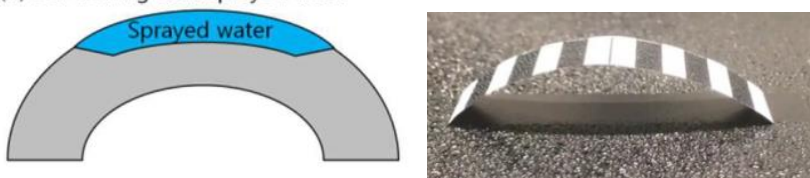

(c) self-unfolding with evaporation
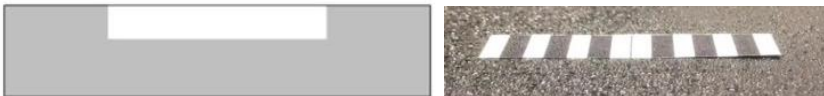

Figure 2: Schematic representations of the device operation principle with photo-images of the self-folding and selfunfolding paper.

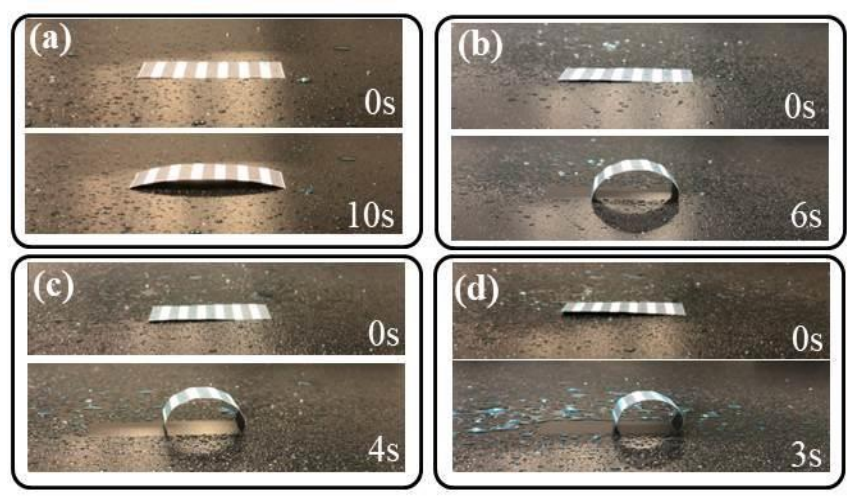

Figure 3: Time courses of our devices' self-folding with different amount of water; (a) 30\% wetting (b) $60 \%$ wetting, (c) $80 \%$ wetting, and (d) $100 \%$ wetting.

move compared to the active layer with the water-based swelling process. This difference in expansion or contraction between the two layers generates localized bending of the paper sheet.

\section{RESULT AND DISCUSSION}

\section{Self-folding papers}

The bilayer of the wax and the hydrophilic channel had drastically different swelling behavior in response to water spraying, spontaneously folding-up and actuating from the 2-D pattern on the paper (Figure 2). The folding angle of the paper increased more rapidly as the more water was introduced (Figure 3 ). With the more water amount, the surface tension of the channel layer increased and thus the higher folding angle could be obtained. With $30 \%$ wetting of the paper channel, the paper did not fold even $10 \mathrm{~s}$ after the water introduction while it rapidly selffolded within 3 seconds under $100 \%$ wetting condition. Furthermore, different 3-D paper structures were created with different wax/channel patterns in paper: (a) half oval, (b) circle, (c) round-edge square, (d) triangle, (e) half-circle, and (f) table (Figure 4). (a) The half oval structure was obtained using a relatively wide wax pattern in the middle and the periodic wax/channel patterns with the same width on the other sides.

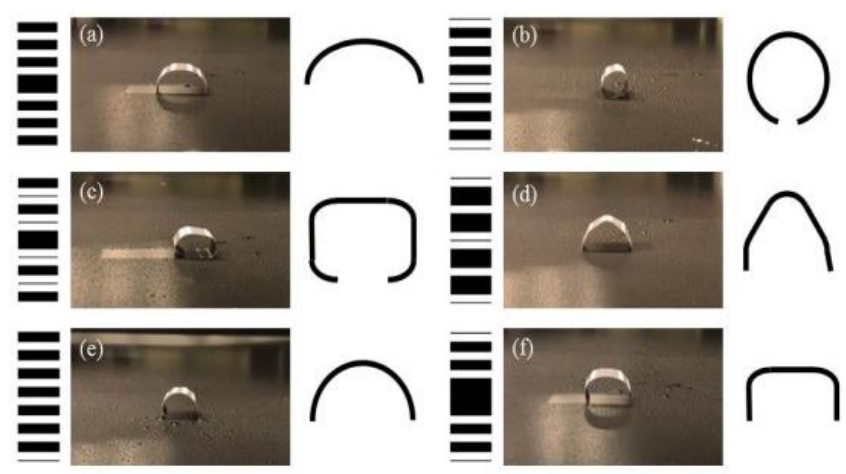

Figure 4: Different deformation based on different wax/channel designs. (a) Half-oval, (b) circle, (c) round-edge square box, (d) tip, (e) half-circle, and (f) table.
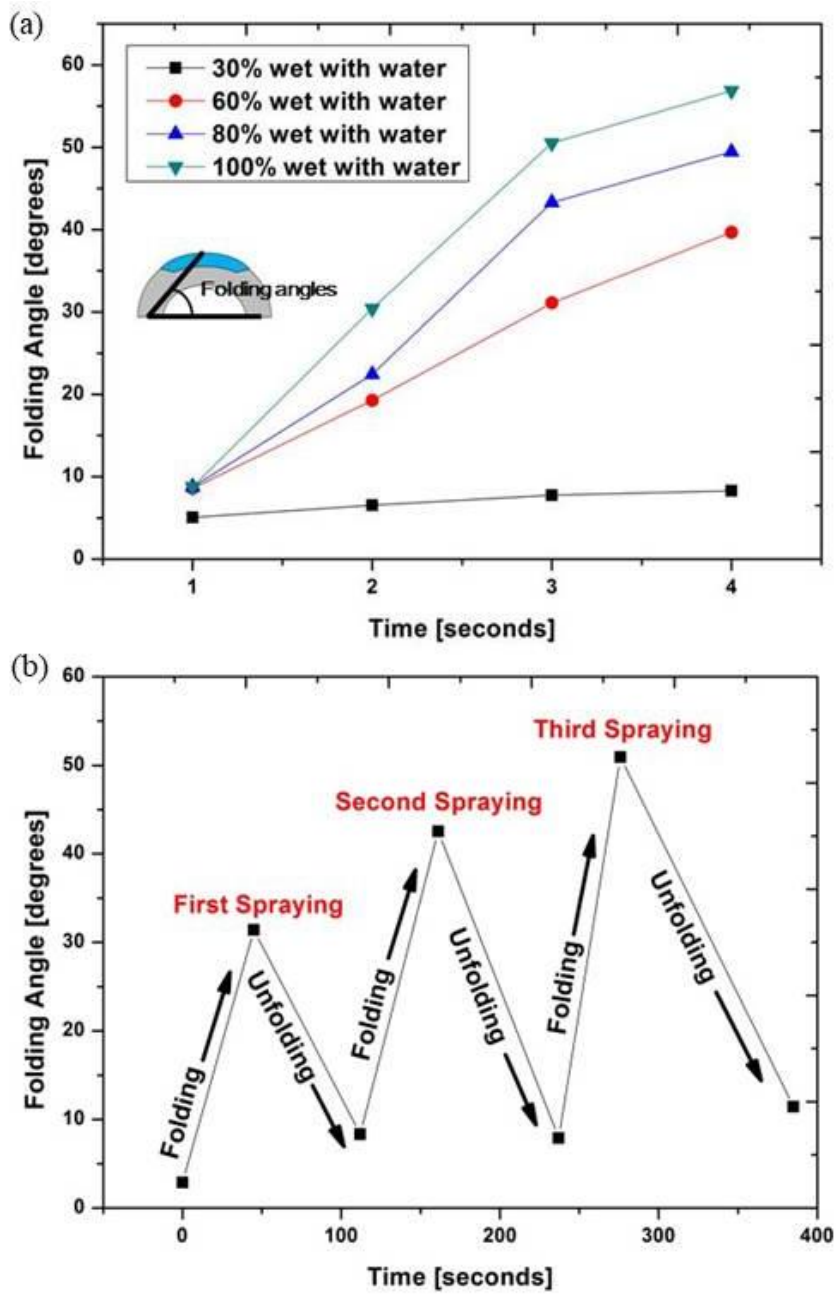

Figure 5: (a) Folding actuation with water. The folding angles increased with the higher wet percentage in paper. (b) Continuous folding/unfolding steps.

(b) The similar wax/channel patterns to the half oval one formed the circle structure except the edge and middle patterns having hydrophilic channels to provide the circular folding. (c) For the round-edge square box, the hydrophilic channel had to be enlarged while thin wax patterns were included within the channel. (d) The 

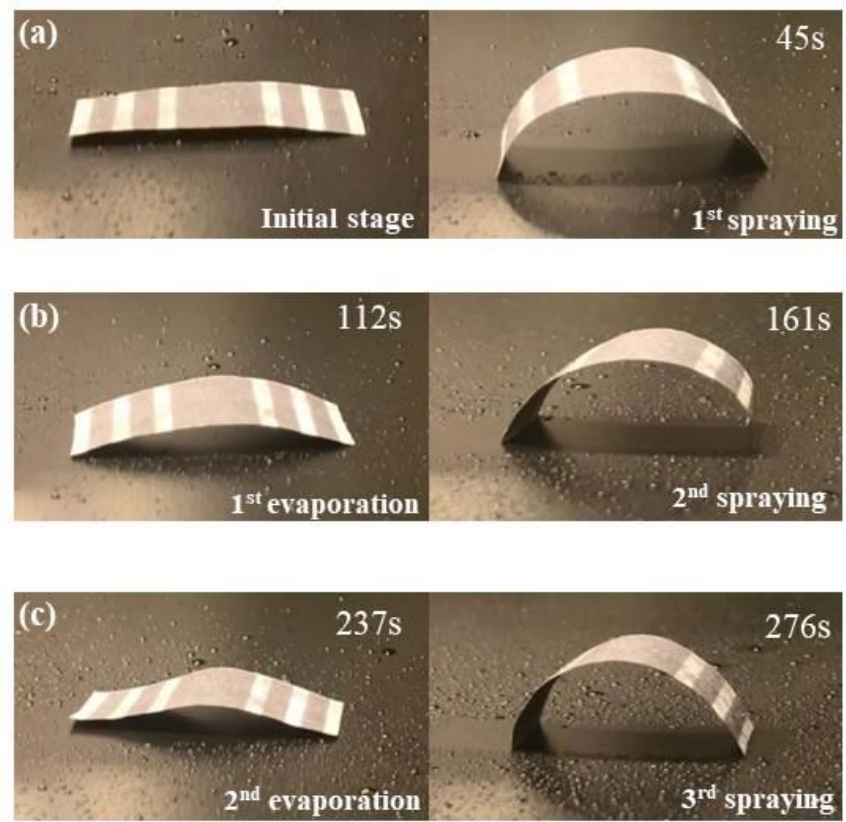

Figure 6: Time courses of continuous folding/unfolding steps. (a) Initial stage $\rightarrow 1^{\text {st }}$ folding, (b) $1^{\text {st }}$ unfolding $\rightarrow 2^{\text {nd }}$ folding, and (c) $2^{\text {nd }}$ unfolding $\rightarrow 3^{\text {rd }}$ folding.

tip structure was formed with the increased widths of the wax patterns. (e) The half-circle structure was developed with the periodic wax/channel patterns with the same width. Finally, (f) the table was acquired with the increased wax width in the middle from the half circle pattern. Controlling shapes of the self-folding paper robots is very important not only to adapt to different environments but also to create different locomotion behaviors. The folding process relied on the water amount sprayed on the paper, demonstrating the folding angles more gradually increased with the higher wet percentage in paper (Figure 5a). At 4 seconds after water spraying, $100 \%$ wetting made a 57 -degree bend while 50-degree, 38-degree, and 7-degree bends were made for $80 \%$, $60 \%$, and $30 \%$ wetting condition, respectively.

\section{Repeated folding and unfolding}

The folded structure can also unfold itself with evaporation (Figure 5b \& Figure 6). Unlike the previous reports [16, 17], our paper with asymmetric wax/channel patterns did not reversely fold. Under repeated swelling and drying processes, the designed paper successfully folded and unfolded. However, it should be noted that the complete unfolding required more time, which did not return to its initial flat stage of the paper. During the repeated folding and unfolding, the folding angle gradually increased mainly because the paper rigidity decreased after multiple mechanical movements.

\section{Paper grippers}

In contrast to robots made from rigid materials, soft robots can gently grip and manipulate irregular/delicate objects [20-22]. These systems provide the unique advantage of inherently safe and adaptive contact with non-planar surroundings, which open a frontier for the next generation wearable and field robots [20]. In this task, we developed a six-legged paper gripper (Figure 7) in which each leg had the wax/channel patterns for the half-circle structure (Figure 4e). We selected the number of the legs and adjusted their lengths to effectively pick up and manipulate a soft
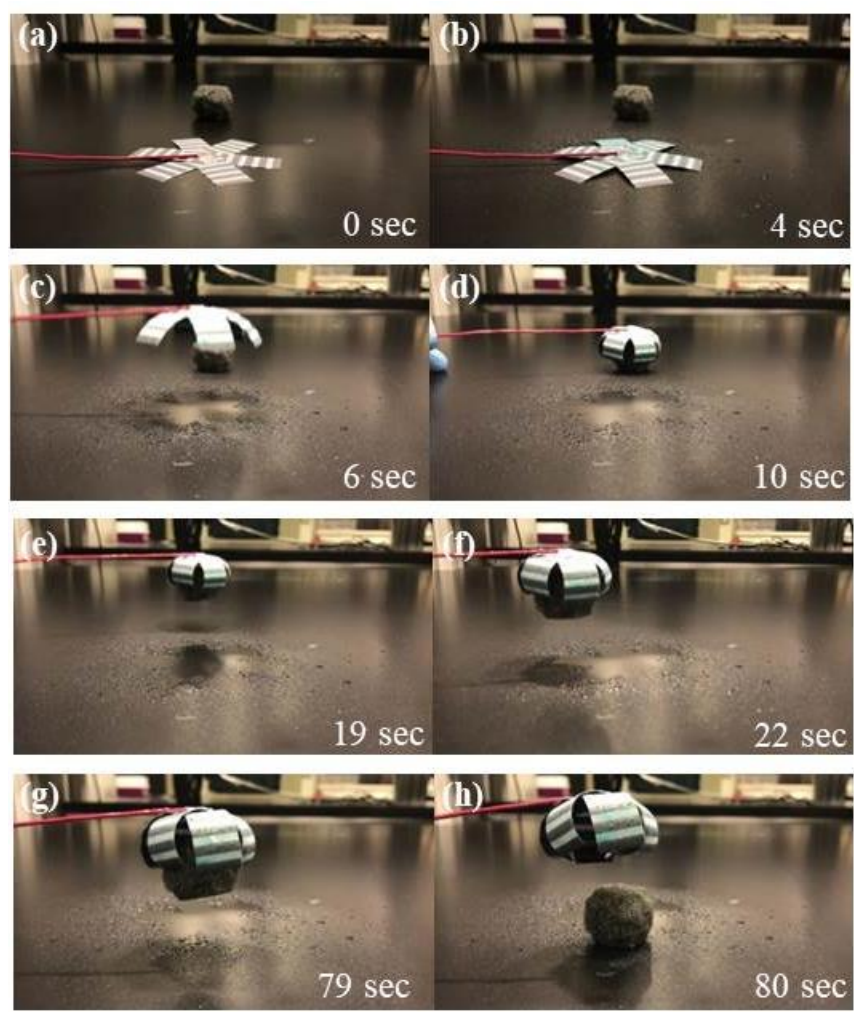

Figure 7: Still frames from a video of the paper gripper performing a manipulation task with an object. In this task, a sponge is grasped, manipulated, and released. Frames at: (a) $0 s$, (b) $4 \mathrm{~s}$, (c) $6 \mathrm{~s}$, (d) 10s, (e) 19s, (f) 22s, (g) 79s, and (h) $80 \mathrm{~s}$.

target object with specific size, weight and shape. Upon the water spraying, each leg self-folded to form a half circle structure. Our paper gripper successfully picked up the soft circular sponge with the same weight as the paper gripper and held it for more than a minute. When the water in the channel evaporated, the gripper released the object.

\section{CONCLUSION}

In this work, we demonstrated an innovative technique to fabricate fluid-driven self-folding papers, and their applications for robotics. The single 2D sheet of papers self-folded into various 3D structures when water was introduced. Folding times and shapes could be controlled by changing the designs of the hydrophilic and hydrophobic patterns and controlling the amount of water sprayed on paper. Furthermore, the paper could fold multiple times when water was re-introduced to the dried hydrophilic channels. The self-folding papers were leveraged to develop the paper gripper with six soft legs, which could pick up a small and low-weight object and hold it for about one minute. We believe that this microfluidic control with paper provides an important strategy to build more powerful and capable selfactuating robots.

\section{ACKNOLOWDGMENT}

This work is supported by NSF (ECCS \#1503462) and the SUNY Binghamton Research Foundation (SE-TAE). 


\section{REFERENCES}

[1] C. Majidi, "Soft Robotics: A Perspective - Current Trends and Prospects for the Future," Soft Robotics, 1, 154, 2014.

[2] S. Kim, C. Laschi, B. Trimmer, "Soft robotics: A bioinspired evolution in robotics," Trends in Biotech., 31, 287, 2013.

[3] G. Haghiashtiani, E. Habtour, S.H. Park, F. Gardea, M.C. McAlpine, "3D printed electrically-driven soft actuators," Extreme Mechanics Letters, 21, 1, 2018.

[4] H. Banerjee, Z.T.H. Tse, H. Ren, "Soft robotics with compliance and adaptation for biomedical applications and forthcoming challenges," International Journal of Robotics and Automation, 33, 69, 2018.

[5] A.W. Feinberg, "Biological Soft Robotics," Annual Review of Biomedical Engineering, 17, 243, 2015.

[6] N. Lu, D. Kim, "Flexible and Stretchable Electronics Paving the Way for Soft Robotics," Soft Robotics, 1, 53, 2014.

[7] T. Arnold, M. Scheutz, "The tactile ethics of soft robotics: Designing wisely for human-robot interaction," Soft Robotics, 4, 81, 2017.

[8] E.A. Peraza-Hernandez, et al., "Origami-inspired active structures: a synthesis and review," Smart Materials and Structures, 23, 094001, 2014.

[9] J.C. Breger, C. Yoon, R. Xiao, H.R. Kwag, M.O. Wang, J.P. Fisher, T.D. Nguyen, D.H. Gracias, "Self-folding thermosmagnetically responsive soft microgrippers," ACS Appl. Mater. Interfaces, 7, 3398, 2015.

[10] L. Hines, K. Petersen, G.Z. Lum, M. Sitti, “Soft actuators for small-scale robotics," Advanced Materials, 29, 1603483, 2017.

[11] S. Sundaram, D.S. Kim, M.A. Baldo, R.C. Hayward, W. Matusik, "3D-printed self-folding electronics," ACS. Appl. Mater. Interfaces, 9, 32290, 2017.

[12] M.M. Hamedi, V.E. Campbell, P. Rothemund, F. Guder, D.C. Christodouleas, J. Bloch, G.M. Whitesides,"Electrically activated paper actuators," Advanced Functional Materials, 26, 2446, 2016.

[13] A.S. Chen, H. Zhu, Y. Li, L. Hu, S. Bergbreiter, "A paperbased electrostatic zipper actuator for printable robots," 2014 IEEE International Conference on Robotics \& Automation, May 31-June 7, 2014, Hong Kong, China, pp.5038-5043.

[14] X. Du, H. Wu, J. Qi, S.Y. Jeong, F. Ni, "Paper-based pneumatic locomotive robot with sticky actuator," MATEC Web of Conferences 42, 03014, 2016.

[15] P. Andersson, R. Forchheimer, P. Tehrani and M. Berggren, "Printable All-Organic electrochromic Active-Matrix displays," Advanced Functional Materials, 17, 3074, 2007.

[16] H. Shigemune, S. Maeda, Y. Hara, U. Koike, S. Hashimoto, "Kirigami robot: making paper robot using desktop cutting plotter and inkjet printer," 2015 IEEE/RSJ International Conference on Intelligent Robot and Systems, Sep. 28 - Oct. 2, 2015, pp.1091-1096.

[17] H. Shigemune, S. Maeda, Y. Hara, N. Hosoya, S. Hashimoto, "Origami robot: a self-folding paper robot with an electrothermal actuator created by printing," IEEE/ASME Transactions on Mechatronics, 21, 2746, 2016.

[18] S. Jeong, S. Lee, C. Choi, J. Kim, C. Lee, "Toward instrument-free digital measurements: a three-dimensional microfluidic device fabricated in a single sheet of paper by double-sided printing and lamination," Lab on a Chip, 15, 1188, 2015.

[19] Y. Gao, S. Choi, "Stepping toward self-powered papertronics: Integrating biobatteries into a single sheet of paper," Advanced Materials Technologies, 2, 1600194, 2017.
[20] X. Zhou, C. Majidi, O.M. O’Reilly, "Soft hands: an analysis of some gripping mechanisms in soft robot design," International Journal of Solids and Structures, 64065, 155, 2015.

[21] J. Zhou, S. Chen, Z. Wang, "A soft-robotic gripper with enhanced object adaptation and grasping reliability," IEEE Robotics and Automation Letters, 2, 2287, 2017.

[22] K. Tai, A. El-Sayed, M. Shahriari, M. Biglarbegian, S. Mahmud, "State of the art robotic grippers and applications," Robotics, 5, 11, 2016.

\section{CONTACT}

*Seokheun Choi, Assistant Professor, SUNY-Binghamton, Email:sechoi@binghamton.edu

Website: http://ws.binghamton.edu/choi 